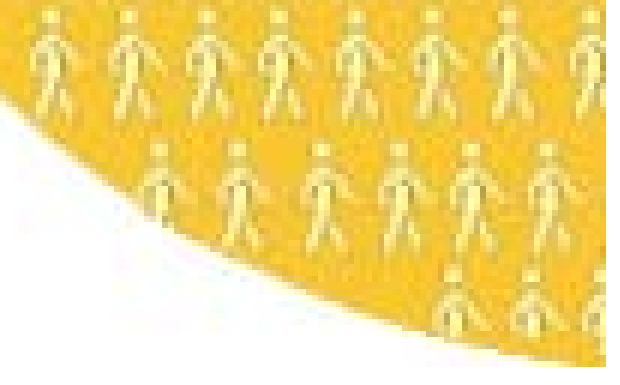

\title{
IDENTIFYING HEDONIC MODELS
}

\author{
Ivar Ekeland \\ James J. Heckman \\ Lars Nesheim
}

THE INSTITUTE FOR FISCAL STUDIES DEPARTMENT OF ECONOMICS, UCL cemmap working paper CWP06/02 


\title{
Identifying Hedonic Models
}

\author{
Ivar Ekeland, James J. Heckman and Lars Nesheim*
}

December, 2001

Economic models for hedonic markets characterize the pricing of bundles of attributes and the demand and supply of these attributes under different assumptions about market structure, preferences and technology. (See Jan Tinbergen, 1956, Sherwin Rosen, 1974 and Dennis Epple, 1987, for contributions to this literature). While the theory is well formulated, and delivers some elegant analytical results, the empirical content of the model is under debate. It is widely believed that hedonic models fit in a single market are fundamentally underidentified and that any empirical content obtained from them is a consequence of arbitrary functional form assumptions.

The problem of identification in hedonic models is a prototype for the identification problem in a variety of economic models in which agents sort on unobservable (to the economist) characteristics: models of monopoly pricing (Michael Mussa and Sherwin Rosen, 1978; Robert Wilson, 1993) and models for taxes and labor supply (James Heckman, 1974). Sorting is an essential feature of econometric models of social interactions. (See William Brock and Steven Durlauf, 2001). In this paper we address the sorting problem in hedonic models. Nesheim (2001) extends this analysis to a model with peer effects.

In this paper we note that commonly used linearization strategies made to simplify estimation and justify the application of instrumental variables methods, produce identification problems. The hedonic model is generically nonlinear. It is the linearization of a fundamentally nonlinear model that produces the form of the identification problem that dominates discussion in the applied literature. Linearity is an arbitrary and misleading functional form when applied to empirical hedonic models.

Our research establishes that even though sorting equilibrium in a single market implies no exclusion restrictions, the hedonic model is generically nonparametrically identified. Instrumental variables and transformation model methods identify economically relevant parameters even 
without exclusion restrictions. Multimarket data, widely viewed as the most powerful source of identification, achieves this result only under implausible assumptions about why hedonic functions vary across markets.

\section{The Hedonic Model}

For specificity, consider a static labor market setting. Firms and workers match on attributes $z$. Matches are 1-1. $P(z)$ is the earnings of a worker who supplies attributes $z$, and $R$ is unearned income. $P(z)$ is the hedonic function. $U(c, z, A, \theta)$ are preferences of workers where $A$ represents preference parameters common across persons and $\theta$ represents preference heterogeneity parameters that differ across people and $c$ is consumption $(c=P(z)+R)$. Given $P(z)$, assumed twice differentiable, we obtain the standard first order and second conditions for a maximum. There is a parallel problem for profit maximizing firms that have technology $F(z ; \nu, B)$ where $\nu$ is a vector of technology parameters that vary across firms, and $B$ is a common technology parameter. Strictly interior solutions are assumed. For simplicity we assume that $\operatorname{dim} \theta=\operatorname{dim} \nu$ where "dim" is dimension. Neither $\theta$ nor $\nu$ are assumed to be observable to the economist.

Under standard assumptions, we may invert the first order conditions to obtain the implicit functions that map unobservables to observables and common parameters, $\theta=\theta\left(z, P_{z}, P(z)+R, A\right)$ and $\nu=\nu\left(z, P_{z}, B\right)$, where $P_{z}$ is the partial derivative of $P(z)$. Substituting into the densities for $\theta$ and $\nu$ we obtain the celebrated Rosen (1974) - Tinbergen (1956) equilibrium condition for hedonic markets that $P(z)$ is the solution to a second order partial differential equation that equates demand and supply densities at each point of $z$ in which a market operates. Under standard conditions, and with sufficient boundary conditions, $P(z)$ is uniquely determined from the equilibrium conditions.

\section{A Linear - Quadratic Example}

Assume preferences are quadratic in $z$ and that $\operatorname{dim}(z)=\operatorname{dim}(\theta)$ :

$$
U(c, z, \theta, A)=R+P(z)+\theta^{\prime} z-\frac{1}{2} z^{\prime} A z
$$

The conditions determining a consumer maximum set $\theta-A z+P_{z}$ to zero and imply that $\left(P_{z z^{\prime}}-A\right)$ is negative definite ${ }^{1}$. On the firm side, assume the production function is quadratic in $z$ and 
$\operatorname{dim}(z)=\operatorname{dim}(\nu)$. Profits are

$$
\Pi(z, \nu, B, P(z))=\nu^{\prime} z-\frac{1}{2} z^{\prime} B z-P(z)
$$

and the conditions determining a firm's optimum set $\nu-B z-P_{z}$ to zero and imply that $-\left(B+P_{z z^{\prime}}\right)$ is negative definite. The distributions of $\nu, \theta$ in the population are normal. The distribution of $\theta$ is $\theta \sim N\left(\mu_{\theta} ; \Sigma_{\theta}\right)$, and the distribution of $\nu$ is $\nu \sim N\left(\mu_{\nu} ; \Sigma_{\nu}\right)$.

The price function induces a density of demand and a density of supply at every location $z$. The equilibrium price function can be found by equating these densities at every point $z$ and solving the resulting differential equation. In the normal-linear-quadratic case, the solution to the problem is quadratic in $z$ :

$$
P(z)=\pi_{0}+\pi_{1}^{\prime} z+\frac{1}{2} z^{\prime} \pi_{2} z
$$

Assuming the price function is quadratic, the first order conditions for the firm are:

$$
\text { Firm: } \nu-B z-\pi_{1}-\pi_{2} z=0
$$

and for the consumer they are:

$$
\text { Consumer: } \theta-A z+\pi_{1}+\pi_{2} z=0 \text {. }
$$

From the second order conditions, $B+\pi_{2}$ and $A-\pi_{2}$ are positive definite. Thus we may solve for $z$ from (1) to obtain $z=\left(B+\pi_{2}\right)^{-1}\left(\nu-\pi_{1}\right)$ and from (2) $z=\left(A-\pi_{2}\right)^{-1}\left(\theta+\pi_{1}\right)$. Note that once we have solved for $\pi_{1}$ and $\pi_{2}$, these latter two equations define the equilibrium matching function linking the characteristics of demanders (1) to those of suppliers (2). For each $z$, this function is

$$
\left(B+\pi_{2}\right)^{-1}\left(\nu-\pi_{1}\right)=\left(A-\pi_{2}\right)^{-1}\left(\theta+\pi_{1}\right)
$$

To characterize equilibrium, we must equate demand and supply. In this linear normal case, all that is required is to equate mean demand to mean supply and the variances of supply to the variances of demand. Rearranging terms, we obtain an explicit expression for $\pi_{1}$ in terms of $A, B, \mu_{\nu}, \mu_{\theta}$ and $\pi_{2}:$ 


$$
\left[\left(A-\pi_{2}\right)^{-1}+\left(B+\pi_{2}\right)^{-1}\right]^{-1}\left[\left(B+\pi_{2}\right)^{-1} \mu_{\nu}-\left(A-\pi_{2}\right)^{-1} \mu_{\theta}\right]=\pi_{1} .
$$

To determine $\pi_{2}$, compute the variances of demand and supply from (1) and (2) respectively to obtain the equilibrium condition:

$$
\left(B+\pi_{2}\right)^{-1} \sum_{\nu}\left(B+\pi_{2}\right)^{-1}=\left(A-\pi_{2}\right)^{-1} \sum_{\theta}\left(A-\pi_{2}\right)^{-1} .
$$

We pin down initial conditions using the restrictions that $U \geq \bar{U}$, a reservation value, and profits are positive $(\Pi \geq 0)$. These imply $\pi_{0}=0$.

\section{Identifying The Model}

Following Rosen (1974), we consider the problem of using data from a single market in which $P(z)$ is available and there are no missing attributes. Using the first order conditions $((1)$ and

(2) in the linear-quadratic-normal example) he proposed a two step method for estimating both preference and technology parameters. He did not consider direct estimation of production, profit or preference functions, a source of information we consider in our companion paper. If there are no missing attributes, one can recover the production function directly from data on inputs and outputs using standard methods. Even if production (or profit) data are available, data on utility are not, so the problem considered by Rosen remains for recovering the parameters of at least one side of the market.

From our discussion of the linear - quadratic - normal case, the parameters $\pi_{1}$ and $\pi_{2}$ do not directly identify either preference or technology parameters except when $\sum_{\theta}=0$ or $\sum_{\nu}=0$ respectively. The pricing function combines parameters in a difficult-to-interpret fashion.

The most direct approach to estimating the hedonic model would be to solve the second order differential equation implied by equilibrium for $P(z)$ in terms of the parameters of preferences, technology and the distributions of tastes and productivity and to jointly estimate the demand functions and supply functions and distributions of preference and technology parameters exploiting all of the information in the equilibrium conditions including data on demand, supply and the pricing function. That approach is computationally complicated and does not transparently deliver identification of the deep structural parameters. 
Rosen suggested an intuitively plausible and computationally simpler two step estimation procedure that has been widely criticized. In step 1 of his procedure, the analyst estimates $P(z)$ from market data. In step 2, the analyst uses first order conditions in conjunction with the marginal prices obtained from step 1 to recover preferences and technology respectively.

In the context of the linear-quadratic example, the first stage would be to estimate pricing function $P(z)$, recover $\pi_{1}$ and $\pi_{2}$, and form the marginal prices and then estimate the curvature parameters of technology, and preferences using (1) and (2) respectively. Specifically, he proposed to estimate $B$ and the mean of $\nu\left(\mu_{\nu}\right)$ from the least squares regression

$$
\widehat{P}_{z}(z)=\hat{\pi}_{1}+\hat{\pi}_{2} z=\mu_{\nu}+B z+\varepsilon_{\nu}
$$

where $\varepsilon_{\nu}=\nu-\mu_{\nu}$, and "^" denotes estimate. A parallel proposal for preferences estimates $A$ and the mean of $\theta\left(\mu_{\theta}\right)$ from the regression

$$
\widehat{P}_{z}(z)=\hat{\pi}_{1}+\hat{\pi}_{2} z=\mu_{\theta}+A z+\varepsilon_{\theta}
$$

where $\varepsilon_{\theta}=\theta-\mu_{\theta}$. We assume that $\mu_{\theta}$ and $\mu_{\nu}$ are functions of regressors $(x)$ and $(y)$ respectively, $\mu_{\theta}(x)$ and $\mu_{\nu}(y)$.

In an influential paper, James Brown and Harvey Rosen (1982) analyze the regression method based on this idea. These papers contain most of the main ideas in the empirical literature on hedonics that emerged from Rosen's paper. They interpret (3) and (4) as linearized approximations to the general first order conditions for the model. The linear-quadratic-normal is the framework in which these approximations are exact.

In this approximation interpretation, the distributions of $\nu$ and $\theta$ are kept in the background. Standard linear econometric methods are applied to identify the parameters of (3) and (4) and connections among the parameters of preferences, technology and the distributions of tastes and productivity are not made explicit. Issues of identification are confused with issues of estimation. Common to an entire genre of empirical economics, this literature focuses on finding "good instruments" and misses basic sources of identification in hedonic models. 
Starting from (3) and (4), Brown and Rosen (1982) make three points which have been reiterated in the subsequent empirical literature.

\section{Point One: Identification Can Only Be Obtained Through Arbitrary Functional Form}

\section{Assumptions}

Since $z$ is on both sides of (3) and (4), by a property of least squares, a regression using the constructed price $\widehat{P}_{z}(z)=\hat{\pi}_{1}+\hat{\pi}_{2} z$ as the dependent variable in (3) or (4) only identifies $\pi_{2}$ even if $\mu_{\nu}$ or $\mu_{\theta}$ are functions of regressors. In general, $\pi_{2}$ does not identify any technology or preference parameter. In the special cases where there is no variation in preference parameters $\theta$ or where there is no dispersion in $\nu, \pi_{2}$ identifies preference $(A)$ or production parameters $(B)$ respectively.

However, if the constructed price is a nonlinear function of $z$, this argument no longer holds. The nonlinear variation in $\widehat{P}_{z}(z)$ gives an added piece of information which can help to identify technology and preference parameters. This identification strategy works because it rules out

collinearity between $z$ and $\widehat{P}_{z}(z)$, but such nonlinearity is widely viewed as an artificial source of identification that is thought to be "arbitrary." In our companion paper, we prove that this nonlinearity is a generic property of equilibrium in the hedonic model.

\section{Point Two: Endogeneity}

Even if such "arbitrary" assumptions are made, so that we can use the nonlinearity in $\widehat{P}_{z}(z)$ to help identify the parameters and circumvent Point One, we still face standard endogeneity problems. $z$ is correlated with $\varepsilon_{\nu}$ and $\varepsilon_{\theta}$ in (3) and (4) respectively. Moreover, exclusion restrictions from the other side of the market cannot be justified. The equilibrium matching condition requires that

$$
\varepsilon_{\nu}=\varepsilon_{\theta}+(A-B) z+\mu_{\theta}(x)-\mu_{\nu}(y)
$$

so that conditional on $z$ there is a functional and statistical dependence connecting $\varepsilon_{\theta}, \varepsilon_{\nu}, z$ and the regressors. ${ }^{2}$ Conditional on $z, \varepsilon_{\nu}, \varepsilon_{\theta}, x$ and $y$ become stochastically dependent even if in the underlying population initially they are mutually independent.

With data from a single market, one is forced to hunt for "clever" instruments with a questionable economic basis. Thus, even if "arbitrary" nonlinearities are invoked, standard instruments may be lacking. In our companion paper we show that the economics of the model guarantees valid 
instruments even though there are no exclusion restrictions.

\section{Point Three: Use of Multimarket Data}

Brown and Rosen (1982), Epple (1987) and Kahn and Lang (1988), change Rosen's problem and consider estimation of the first order conditions using multimarket data either across regions, or across time in the same region. The motivation for this approach is that if preferences, technology, and the distributions of tastes and productivities are the same across markets but for some unspecified reason price functions are not, variation in the $P_{z}(z)$ across markets serves to identify preferences and technology. This source of identification is viewed as being more robust.

The problem with this identification strategy is that it is logically inconsistent. If preferences, technology, and the distributions of tastes and productivities are the same across markets, equilibrium price functions must be as well. The strategy is apparently more robust because it is vague about the source of variation that makes price functions differ when preferences, technology, and the distributions of tastes and technology are common across markets. Under suitable restrictions on preferences and technology, this approach can be used to identify the preferences or technology on one side of the market. If preferences are stable and the distributions of preferences across markets are stable, but technologies are different for exogenous reasons, then multimarket variation shifts the hedonic function against stable preferences and identifies preference parameters. Switching the roles of technology and preferences, multimarket data identifies technology and the distribution of technology parameters. If common preference and technology parameters are identical across markets, but heterogeneity distributions are different across markets, then preference and technology parameters and the heterogeneity distributions can be identified.

\section{Using All Of The Economics of The Model}

These criticisms are symptoms of a deeper problem: all of the economic content of the hedonic model is not being exploited. We argue that when it is exploited, the model is generically identified even within a single market without having to invoke arbitrary functional forms. We develop this point formally in our companion paper. Here we develop the intuition for it using the linearquadratic model. 
Consider all of the economic implications of the linear-quadratic model - not just the first order conditions (1) and (2). Any reasonable specification of the model requires that profits be non-negative, that utilities exceed threshold reservation values and that firm marginal products be non-negative while marginal utilities of consumers for disamenities be non-positive. Adopting all of these restrictions eliminates Point One within the linear-quadratic example.

The linear-quadratic-normal model results in an equilibrium with a linear marginal price function. This equilibrium produces an econometric system that is not identified. (Brown-Rosen Point One). In this example, it would be arbitrary and incorrect to impose that the marginal price function is nonlinear. However, the linear-quadratic model in Section II is very special. It belongs to a very small class of models that produce an equilibrium marginal price function that is linear. In our companion paper, we prove as a special case of a more general theorem that there is an open dense set of models surrounding the linear-quadratic models of Section II that do not produce linear marginal price functions. In these models, it is not arbitrary to impose nonlinear marginal price functions.

The normal-linear-quadratic example has a number of peculiarities. From (1) and (2), it is evident that marginal products can become negative, and marginal disutilities of labor $(z)$ can become positive. Nothing restricts marginal prices to be non-negative or for the demands or supplies of $z$ to be non-negative.

To see how fragile Point One is, suppose that we perturb a scalar version of the linear quadratic model to have non-normal $\theta$ and $\nu$. The Figure shows the price functions for two cases. The parameter values generating the Figure are given at the base. The first case is for $\nu_{1}$ and $\theta_{1}$ normally distributed ( $\lambda=1$; see the notes at the base of the Figure). The second case is for $\nu_{1}$ and $\theta_{1}$ distributed as a mixture of normals with weights $\lambda=.999$ (on the original component which produced the straight line) and $1-\lambda=.001$ (on the other component reported at the base of the Figure). With this minor perturbation, the price function becomes highly nonlinear. The second derivative of the price function is far from zero. A small dose of nonnormality produces a highly nonlinear price function, and undercuts Brown-Rosen Point One. 
These figures also reveal unattractive properties of the linear-quadratic model. Negative and positive quantities of $z$ are demanded and supplied and marginal prices are negative for a large portion of the population. In our companion paper, we consider an example where marginal prices are positive. In that case, marginal prices are nonlinear and positive and only positive quantities of the amenity are demanded and supplied. By imposing economically plausible restrictions, BrownRosen Point One is shown to be less cogent. In our companion paper, we show that these examples are generic. They apply to a broad class of models with separable first order conditions and not just linear-quadratic.

Even though Point One is non-generic, Point Two remains. There are apparently no valid instruments for $z$ on the right hand sides of (3) and (4). A strategy needs to be found to deal with the endogeneity of $z$ without exclusion restrictions. In our companion paper, we discuss two such strategies based on transformation models and instrumental variables models and present identification results for a general separable model with a single characteristic with no arbitrary functional form restrictions or distributional assumptions and establish that the hedonic model is generically identified from data from a single market. Intuitively, even though there are no exclusion restrictions, instrumental variables is a valid estimator in a single market. In the context of equations (3) and (4) one can show that $E(z \mid x)$ and $E(z \mid y)$ are not collinear with $\mu_{\theta}(x)$ and $\mu_{\nu}(y)$ respectively. Thus $x$ and $y$ are valid instruments (for supply and demand equations, respectively) even though they are not excluded from their respective equations. See the proof in our companion paper. 
Brock, William and Steve Durlauf. "Interactions Based Models," in J. Heckman and E. Leamer, editors, Handbook of Econometrics, Vol. 5. Amsterdam: North Holland, 2001, pp. 3297-3380.

Brown, James and Harvey Rosen. "On the Estimation of Structural Hedonic Price Models. Econometrica, $1982, \underline{50}$, pp. 765-769.

Ekeland, Ivar, James J. Heckman and Lars Nesheim. "Identification and Estimation of Hedonic Models", University of Chicago, May 2001 revised November, 2001.

Epple, Dennis. "Hedonic Prices and Implicit Markets: Estimating Demand and Supply Functions for Differentiated Products. Journal of Political Economy, 1987, 95 pp. 59-80.

Heckman, James. "The Effect of Day Care Programs on Women's Work Effort." Journal of Political Economy, 1974, 82, pp 491-517.

Kahn, Shulamit and Kevin Lang. "Efficient Estimation of Structural Hedonic Systems," International Economic Review $\underline{29}$ (1988): 157-166.

Mussa, Michael and Sherwin Rosen. "Monopoly and Product Quality."

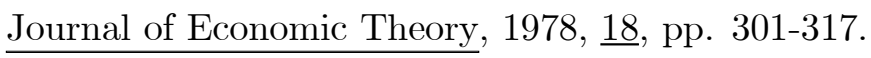

Nesheim, Lars, "Equilibrium Sorting With Heterogeneous Agents: Theory and Empirical Implications," unpublished paper, UCL, London, 2001.

Rosen, Sherwin. "Hedonic Prices and Implicit Markets: Product Differentiation in Pure Competition." Journal of Political Economy, 1974, $\underline{82}$, pp. 34-55.

Tinbergen, Jan. "On The Theory of Income Distribution." Weltwirtschaftliches Archiv, 1956, $\underline{77}$, pp. 155-73.

Wilson, Robert. Nonlinear Pricing. Oxford University Press 1993. 
*This research was supported by NSF 00-99195 and a grant from the American Bar Foundation. Ekeland is at the University of Paris, Dauphine; Email: (ekeland@dauphine.fr), Heckman is in the Department of Economics at the University of Chicago, 1126 E. 59th Street, Chicago, IL 60637, Telephone: (773) 702-0634, Fax (773) 702-8490, Email: jjh@uchicago.edu. Nesheim is at the Institute for Fiscal Studies and the Department of Economics at UCL, London, England, Email: (l.nesheim@ucl.ac.uk ).

${ }^{1}$ The model in this example was first analyzed by Tinbergen (1956) and has previously been used by Epple (1987).

${ }^{2}$ Epple (1987), and Kahn and Lang (1988) stress this point. 
Figure 1

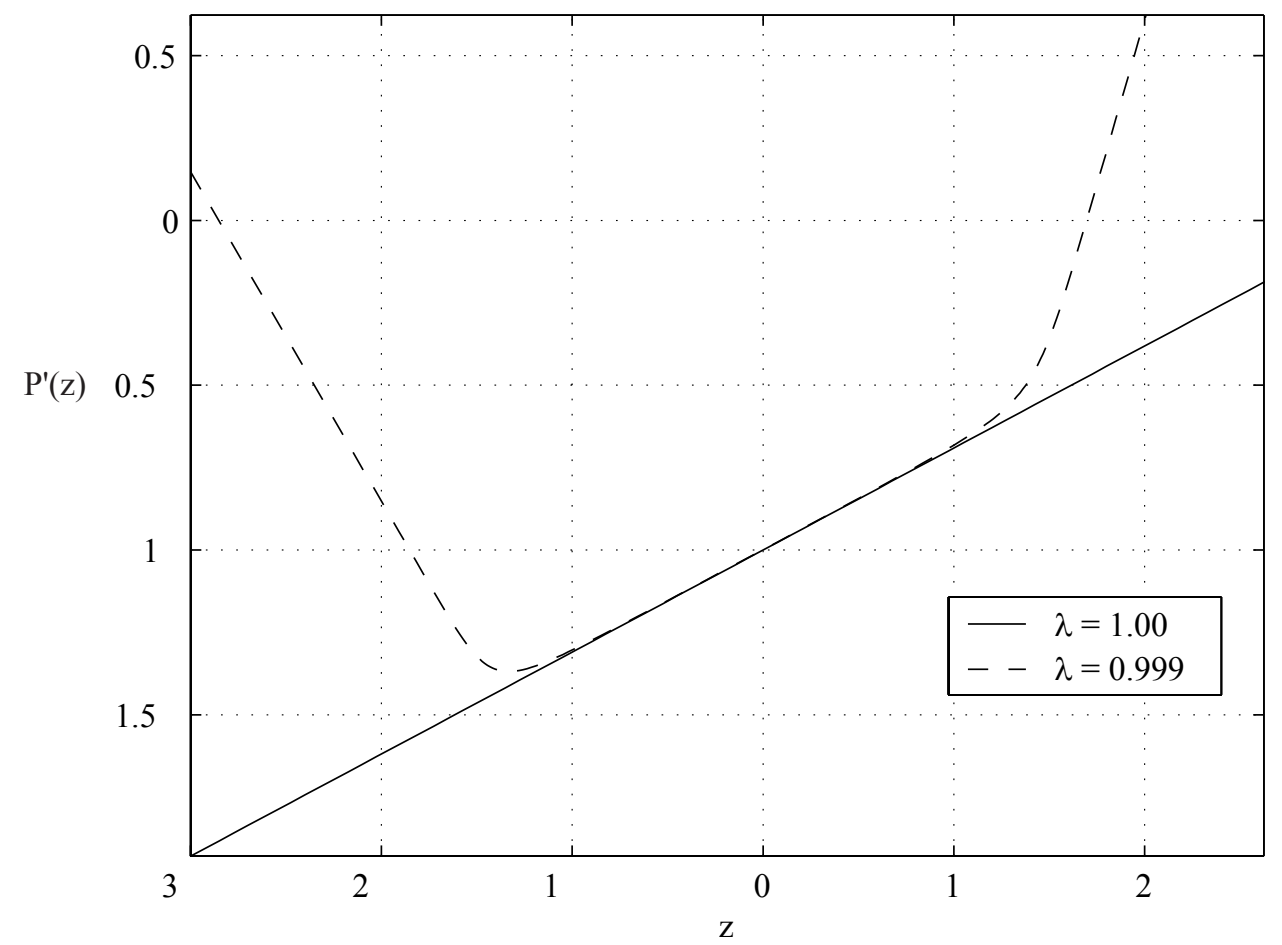


Figure 1

Slope of Price Function

Legend:

$a=2, b=1$

— Normal Linear Quadratic Model

- . - Mixture of Models

Normal Linear Quadratic Model (Tinbergen)

$\mu_{v_{1}}=-1, \sigma_{v_{1}}^{2}=.3, \mu_{\theta_{1}}=.3, \sigma_{\theta_{1}}^{2}=.5$

Mixture of Normals Model

$\lambda=.999$ on Tinbergen Model components and $\lambda=.001$ on $\mu_{v_{1}}=-1, \sigma_{v_{1}}^{2}=.3, \mu_{\theta_{1}}=.3, \sigma_{\theta_{1}}^{2}=.5$ 